\title{
1 Reverse microemulsion synthesis of layered gadolinium hydroxide
}

2

3

4 Yadong Xu, ${ }^{a}$ Jugal Suthar, ${ }^{a}$ Raphael Egbu, ${ }^{a}$ Andrew J. Weston, ${ }^{a}$ Andrew M. Fogg, ${ }^{\text {b* }}$ and Gareth R. 5

6

$8{ }^{\mathrm{b}}$ Department of Chemical Engineering, University of Chester, Thornton Science Park, Cheshire, $\mathrm{CH} 2$ 9

10 * Authors for correspondence. Email: a.fogg@chester.ac.uk (AMF); g.williams@ucl.ac.uk (GRW). Tel: 11

12

13

\section{Abstract}

15A reverse microemulsion approach has been explored for the synthesis of layered gadolinium 16hydroxide (LGdH) nanoparticles in this work. This method uses oleylamine as a multifunctional agent, 17acting as surfactant, oil phase and base. 1-butanol is additionally used as a co-surfactant. A 18systematic study of the key reaction parameters was undertaken, including the volume ratio of 19surfactant (oleylamine) to water, the reaction time, synthesis temperature, and the amount of co20surfactant (1-butanol) added. It proved possible to obtain pristine LGdH materials at temperatures of $21120^{\circ} \mathrm{C}$ or below with an oleylamine : water ratio of 1:4. Using larger amounts of surfactant or higher 22temperatures caused the formation of $\mathrm{Gd}(\mathrm{OH})_{3}$, either as the sole product or as a major impurity 23phase. The LGdH particles produced have sizes of ca. $200 \mathrm{~nm}$, with this size being largely 24independent of temperature or reaction time. Adjusting the amount of 1-butanol co-surfactant 25added permits the size to be varied between 200 and $300 \mathrm{~nm}$.

\section{Keywords}

27Layered gadolinium hydroxide; reverse microemulsion; nanoparticles; oleylamine 


\section{Introduction}

30lon-exchangeable layered materials have attracted widespread attention for a broad range of 31applications. Materials able to exchange both cations and anions are known [1], with the latter 32 having been more widely studied $[2,3]$. In both cases, the incorporation of guest ions into the 33interlayer space of the host materials can have a number of benefits: for instance, the stability of 34guest species can be improved by intercalation $[4,5]$.

35

360ne family of materials which has been particularly extensively studied is the layered double 37hydroxides (LDHs) [2]. LDHs comprise positively-charged mixed-metal hydroxide layers, with charge38balancing anions in the interlayer region [2]. A large variety of inorganic and organic anions can be 39incorporated into their interlayer space by ion-exchange reactions [6-8]. This rich intercalation 40chemistry has been extensively exploited: LDHs have been investigated for applications such as flame 41retardants [9, 10], catalysts and catalyst precursors [11], $\mathrm{CO}_{2}$ adsorbents [12-16], cement additives 42[17] and drug delivery systems [7, 18-20]. Bioactive molecules have been intercalated into the 43interlayer space of LDHs on a number of occasions. Examples include non-steroidal anti44inflammatory drugs such as naproxen, diclofenac, gemfibrozil, ibuprofen and 2-propylpenpenoic acid 45[7, 19]. There are also reports of the encapsulation of anticancer drugs (e.g. 5-fluorouracil and 46methotrexate) [21, 22]. LDH-drug intercalates have been found to lead to sustained drug release 47 profiles and reduced side effects compared to the free drug $[7,19]$.

48

49Beyond LDHs, there exists a range of alternative layered materials capable of anion exchange. These 50include the recently reported layered rare-earth hydroxides (LRHs). There are a range of LRHs 51possible, but those capable of anion exchange have the general formula $\left[\mathrm{R}_{2}(\mathrm{OH})_{5}\right]^{+}\left(\mathrm{A}^{\mathrm{n}-}\right)_{1 / \mathrm{n}} \cdot \mathrm{yH}_{2} \mathrm{O}$ $52\left(\right.$ where $\mathrm{R}=\mathrm{Ln}^{3+}, \mathrm{A}^{\mathrm{n}-}=$ an anion, and $\left.1 \leq y \leq 2\right)$. LRHs contain lanthanide cations and hydroxide ions in 53their positively charged layers, and charge-balancing anions in the interlayer region [23-25]. Typical 54examples include $\left[\mathrm{Gd}_{2}(\mathrm{OH})_{5}\right] \mathrm{Cl} \cdot 1.5 \mathrm{H}_{2} \mathrm{O}$ and $\left[\mathrm{Yb}_{2}(\mathrm{OH})_{5}\right] \mathrm{Cl} \cdot 1.5 \mathrm{H}_{2} \mathrm{O}$ [3]. The inorganic anions typically 55 present in the gallery of LRHs immediately after synthesis can be readily replaced by other inorganic 56or organic species such as azamacrocyclic crown ether [26], or amino acids [27].

57

58LRHs could thus be potent alternatives to LDHs for use as, for instance, drug delivery systems. 59Moreover, LRHs possess the magnetic and fluorescent properties of the rare-earth metals they 60contain, which could give additional benefits. The combination of ion exchange intercalation 61chemistry and rare earth elements in the layers can lead to integrated materials with many 62applications in medical science [28, 29], catalysis [30], separation science [26], sensor technologies 
63[31], and luminescence devices [5, 27, 32-40]. Some studies have focused on incorporating 64 sensitizers or quenchers to tune the colour emission of LRH hybrids, for instance [3, 5, 27].

65

66Magnetic resonance imaging (MRI) is a technique widely used in biomedical imaging. It is popular in 67part because it does not use radioactive agents or high-energy electromagnetic waves, and has high 68spatial and temporal resolution [41]. To obtain good quality images, however, the patient must be 69administered what is termed a "contrast agent", a chemical entity used to enhance the quality of the 70images obtained and permit accurate diagnoses. Commercial contrast agents are commonly based 71on $\mathrm{Gd}^{3+}$. This is because the electronic relaxation time of $\mathrm{Gd}^{3+}$ is very long and it has a high number of 72unpaired electrons, which means that it can enhance both the longitudinal $\left(r_{1}\right)$ and transverse $\left(r_{2}\right)$ 73relaxation times of water protons [42]. Free $\mathrm{Gd}^{3+}$ is extremely toxic, and thus the agents used in the 74clinic are based on chelation complexes designed to ensure that the Gd present remains complexed 75at all times. An alternative route to preclude free $\mathrm{Gd}^{3+}$ getting into solution is to incorporate it into an 76inorganic matrix, and hence layered gadolinium hydroxides (LGdHs) might be viable contrast agents 77[43]. The potential of LGdHs in this regard has been explored in several reports [28, 29, 43, 44], and 78the results obtained are promising. The LGdH matrix has also attracted a little attention for use as a 79drug delivery system, with the intercalation of several pharmaceutically active molecules including 80antibiotics, amino acids, and microRNA [43, 45].

81

82Control of particle size can be extremely important to achieve the desired results in vivo, and for this 83reason much attention has been paid in particular to the production of nanoscale materials, which 84have been explored in many fields [46]. The size of LDH particles has been shown to play an 85important role in their interactions with cells, for instance [47]. Therefore, controlling the particle 86size of LGdH will be important to ensure uptake by cells, and thus to improve its performance in MRI 87applications. However, little effort has been applied to the synthesis of LRH nanoparticles to date. 88

890 ne route to control particle size is the use of reverse microemulsion systems. These comprise water 90droplets dispersed in an oil continuous phase; when materials are grown from an aqueous solution, 91performing the reaction in such systems means that the particle size is controlled by the size of the 92droplets, so long as that the emulsion is stable and there is no coalescence. This approach has been 93found to lead to high degrees of control over particle size, morphology, geometry, and surface area 94[46], and microemulsion systems have emerged as an effective approach to synthesize nanomaterials 95such as metallic catalysts [48], semiconductors [49], ceramics [50], and silica [51]. Here, we apply this 96approach for the first time to the synthesis of nanosized LGdH materials, aiming to produce particles 
97with sizes suitable for cellular uptake. We report the synthesis of LGdH nanoparticles using a method 98in which oleylamine acts as oil phase, base and surfactant, permitting an extremely simple 99microemulsion formulation to be employed.

100

\section{$1012 \quad$ Experimental}

\subsection{Materials}

103Oleylamine and 1-butanol were purchased from Sigma-Aldrich (Gillingham, UK), while gadolinium 104chloride hexahydrate was supplied by Alfa Aesar (Heysham, UK). All water used was deionized, and 105all other chemicals were of analytical grade and used without further purification.

\subsection{Methods}

\subsubsection{General protocol}

109A novel reverse microemulsion method which employs oleylamine as oil phase, base and surfactant 110was developed in 2012 to synthesize LDHs [47]. Experiments were carried out following this method 111 with minor modifications. A $0.5 \mathrm{M}$ solution of $\mathrm{GdCl}_{3} \cdot 6 \mathrm{H}_{2} \mathrm{O}$ in deionized water was first prepared, and 112to this a mixture of oleylamine and 1-butanol was added dropwise with vigorous stirring. After 10 113 min of constant stirring, the resultant mixture was transferred to a Telfon-lined stainless steel 114autoclave $(23 \mathrm{~mL})$ and treated hydrothermally. The resulting precipitates were collected by 115 centrifugation, washed with a mixture of water and ethanol $(1: 1, \mathrm{v} / \mathrm{v})$, and dried at $40{ }^{\circ} \mathrm{C}$ for one day. 116

\subsubsection{Optimization}

118A detailed optimization process was undertaken in this work. First, since the volume ratio of 119surfactant to water is known to be a determining factor for particle size [47, 52], a range of 120oleylamine : water ratios were explored (4:1, 3:2, 1:3 and 1:4). Specifically, 8, 6, 2.5 or $2 \mathrm{~mL}$ of 121oleylamine was first combined with $5 \mathrm{~mL}$ of 1-butanol. The resultant mixtures were added to 2, 4, 7.5 122or $8 \mathrm{~mL}$ of a $0.5 \mathrm{M} \mathrm{Gd}$ chloride solution. The total volume of oleylamine and water was kept at $10 \mathrm{~mL}$, 123and the total solution volume in the autoclave was $15 \mathrm{~mL}$. Hydrothermal treatment was undertaken 124 at $120^{\circ} \mathrm{C}$ for $18 \mathrm{~h}$. The resulting samples are denoted as LGdH-O $m-W n$ ( $m=$ volume of oleylamine, $n=$ 125 volume of water).

126

127Second, the temperature was optimized. With an increase in temperature in a hydrothermal process, 128the particle size of the product tends to be larger [51]; however, high temperatures could also lead to 129potential degradation or phase transformation. The surfactant to water volume ratio was set to $1: 4$, 130and each reaction mixture comprised $2 \mathrm{~mL}$ oleylamine, $5 \mathrm{~mL}$ 1-butanol, and $8 \mathrm{~mL}$ Gd chloride 
131solution. Hydrothermal treatments were carried out at temperatures ranging from $90^{\circ} \mathrm{C}$ to $150^{\circ} \mathrm{C}$ for $13218 \mathrm{~h}$. The products are denoted LGdH- $90^{\circ} \mathrm{C}, \mathrm{LGdH}-120^{\circ} \mathrm{C}$, and LGdH-150 $\mathrm{C}$.

133Third, the aging duration is another factor which could determine the particle size: the longer the 134 crystals grow, the larger they will be. A volume ratio of surfactant to water of 1:4 was used, and again 135each reaction mixture comprised $2 \mathrm{~mL}$ oleylamine, $5 \mathrm{~mL}$ 1-butanol, and $8 \mathrm{~mL} \mathrm{Gd}$ chloride solution. $136 \mathrm{LGdH}$ nanoparticles were prepared with hydrothermal treatment at $12 \mathrm{~h}, 18 \mathrm{~h}$ or $24 \mathrm{~h}$ at $120^{\circ} \mathrm{C}$. The 137products were named LGdH-12h, LGdH-18h, and LGdH-24h.

138Finally, the co-surfactant amount in water-in-oil microemulsion systems has been reported to have 139an important effect on particle growth, with nanoparticle size rising with an increasing amount of co140surfactant [51]. Hence, the volume of 1-butanol was varied from 3 to $9 \mathrm{~mL}$. The ratio of surfactant to 141water was fixed at 1:4, so each reaction mixture comprised $2 \mathrm{~mL}$ of oleylamine, $8 \mathrm{~mL}$ of Gd chloride 142solution, and between 3 and $9 \mathrm{~mL}$ of 1-butanol. Experiments were carried out at $120^{\circ} \mathrm{C}$ for $12 \mathrm{~h}$. The 143materials obtained were designated LGdH-3mL, LGdH-5mL, LGdH-7mL and LGdH-9mL.

\subsection{Characterization}

\subsubsection{X-ray diffraction (XRD)}

146Power XRD patterns were recorded over the $2 \theta$ range from 3 to $45^{\circ}$ on a Rigaku MiniFlex 600 147diffractometer (Tokyo, Japan), using Cu Ka radiation $(\lambda=1.5418 \AA$ ) at $40 \mathrm{kV}$ and $15 \mathrm{~mA}$.

\subsubsection{Fourier transform infrared (FTIR) spectroscopy}

149Infrared spectra were obtained using a Spectrum 100 FTIR spectrometer (Perkin Elmer, Waltham, 150MA, USA) over the range $650-4000 \mathrm{~cm}^{-1}$ with a resolution of $2 \mathrm{~cm}^{-1}$.

\subsubsection{Dynamic light scattering (DLS)}

152Dynamic light scattering measurements were performed on a Zetasizer Nano ZS instrument (Malvern 153Instruments, Malvern, UK). 1-2 mg of LGdH nanoparticles was suspended in ethanol and sonicated 154 for ca. 15 min prior to measurements.

\subsubsection{Transmission electron microscopy (TEM)}

156LGdH nanoparticles $(5 \mathrm{mg})$ were dispersed in ethanol $(1 \mathrm{~mL})$, followed by 20 minutes of sonication. A 157 few drops of each suspension were then placed onto a lacey carbon coated copper grid, and ethanol 
158allowed to evaporate. TEM was performed using a FEI CM120 Bio Twin microscope (Philips, 159Amsterdam, Netherlands) with an accelerating voltage of $120 \mathrm{kV}$.

160

\section{Results}

162The synthesis of LGdH nanoparticles was first attempted using a previously explored protocol in 163which Triton X-100, 2,2,4-trimethylpentane and 1-butanol act as surfactant, oil phase and co164surfactant [53]. However, this method was time-consuming (3 days) and suffered from low yields. In 165addition, the obtained nanoparticles were of very low crystallinity (ascribed to stacking defects), and 166had a wide range of sizes (from tens of nanometers to several micrometers). Replacing 1-butanol 167with propan-2-ol had no significant effect on the findings. This route was thus deemed unsuitable. 168We then turned to an alternative literature protocol. Wang and O'Hare [47] have reported an 169approach using oleylamine (Figure 1) as an multi-functional agent which acts as the oil phase, base, 170and surfactant simultaneously. Alkaline conditions are provided as a result of the amine group, and 171the amine head group along with its alkyl chain tail acts as a surfactant. This leads to the formation 172of reverse micelles in a water-in-oil system. 1-butanol was also added as a co-surfactant, separating 173the oleylamine units and reducing the repulsive forces between the protonated head groups [47]. 174Wang and O'Hare's oleylamine method was systematically explored for the synthesis of LGdH in this 175work.

176

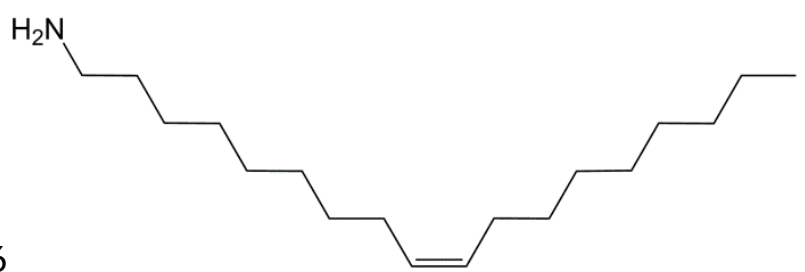

177Figure 1: The chemical structure of oleylamine.

178

\subsection{Effect of surfactant to water ratio}

180The first reaction parameter to be investigated was the ratio of surfactant to water in the system. A 181series of emulsions were prepared as detailed in . 
183Table 1: Details of reactions performed with different ratios of oleylamine to water. All were performed at $120^{\circ} \mathrm{C}$ for $18 \mathrm{~h}$, 184 with $5 \mathrm{~mL}$ of 1-butanol added.

\begin{tabular}{|c|c|c|c|}
\hline Sample ID & $\begin{array}{l}\text { Oleylamine volume } \\
(\mathrm{mL})\end{array}$ & $\begin{array}{ll}\text { Aqueous } & \text { solution } \\
\text { volume }(\mathrm{mL}) & \end{array}$ & $\begin{array}{l}\text { Oleylamine/water volume } \\
\text { ratio }\end{array}$ \\
\hline LGdH-O8-W2 & 8 & 2 & $4: 1$ \\
\hline LGdH-O6-W4 & 6 & 4 & $3: 2$ \\
\hline LGdH-02.5-W7.5 & 2.5 & 7.5 & $1: 3$ \\
\hline LGdH-O2-W8 & 2 & 8 & $1: 4$ \\
\hline
\end{tabular}

185The powder XRD patterns of the products obtained are presented in Figure 2. Phase-pure LGdH 186nanoparticles could be synthesized only with a 1:4 volume ratio of oleylamine to water. At ratios 187below this, the diffraction patterns very closely resemble that of $\mathrm{Gd}(\mathrm{OH})_{3}$ (JCPDS \#38-1042). At ratios 188of 4:1 and 3:2, no lamellar (0OI) reflections at all were observed in the XRD data. At a volume ratio of 1891:3, a weak reflection (marked with an arrow in Figure 2) at ca. $10.5^{\circ}$ was observed, which 190corresponds to the (001) Bragg reflection of LGdH and indicates the presence of a small amount of 191the $\mathrm{LRH}$ alongside the $\mathrm{Gd}(\mathrm{OH})_{3}$ majority product. The pattern obtained for sample LGdH-O2-W8, with 192a 1:4 ratio of surfactant to water, matches literature data for chloride intercalated LGdH (LGdH-Cl) 193[54-56]. The position of the (001) Bragg reflection corresponds to an interlayer spacing of $8.48 \AA$, in 194 excellent agreement with previously reported values for chloride intercalates $[28,56]$.

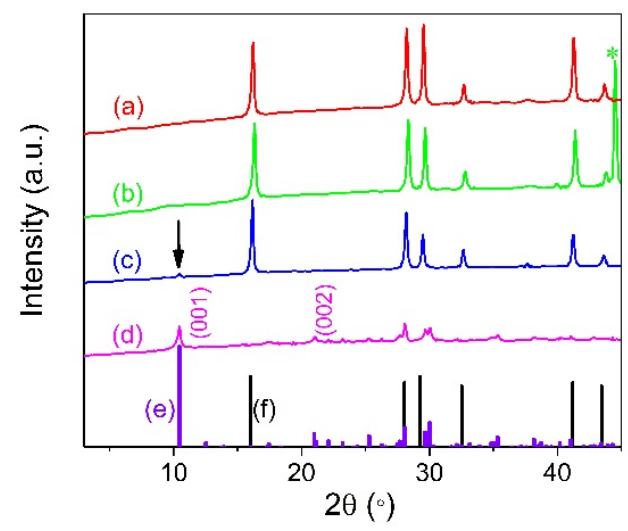

195

196Figure 2: Powder X-ray diffraction patterns of LGdH nanoparticles prepared with different volume ratios of surfactant to 197water: (a) LGdH-O8-W2; (b) LGdH-O6-W4; (c) LGdH-O2.5-W7.5; (d) LGdH-O2-W8. The calculated reflection positions are 198also shown for (e, purple) LGdH-Cl [56] and (f, black) Gd(OH) ${ }_{3}$ [JCPDS \#38-1042]. The Bragg reflection marked * corresponds 199 to the sample holder.

200 


\subsection{Effect of temperature}

203Data for samples prepared at different temperatures with a 1:4 surfactant to water ratio are given in 204Figure 3.

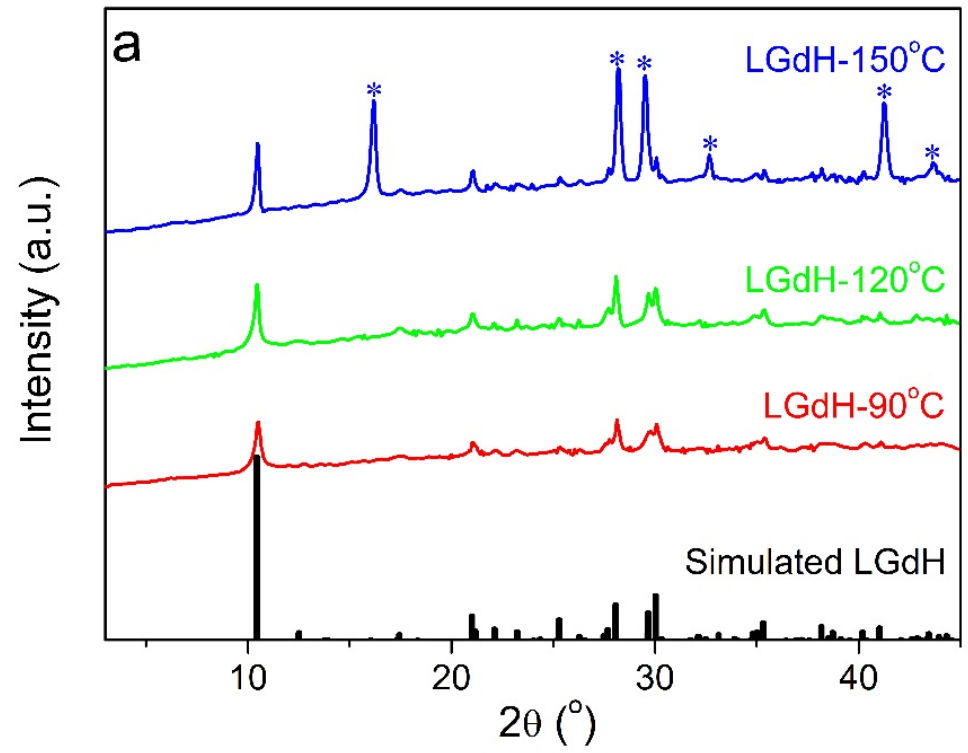

205

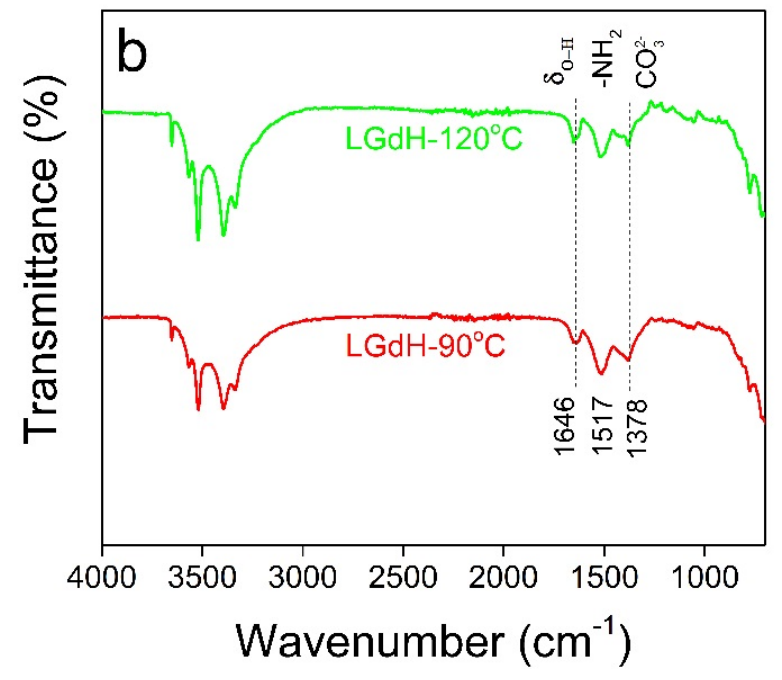

207Figure 3: Experimental data for syntheses conducted at 90,120 , and $150^{\circ} \mathrm{C}$. (a) XRD patterns; and, (b) IR spectra. 208Reflections marked with * in (a) arise from $\mathrm{Gd}(\mathrm{OH})_{3}$.

210The materials generated at 90,120 and $150^{\circ} \mathrm{C}$ all present basal (00I) reflections (Figure 3), indicative 211of an interlayer spacing of $8.42 \AA$. Significant amounts of $\mathrm{Gd}(\mathrm{OH})_{3}$ can be seen in the sample 212 synthesized at $150^{\circ} \mathrm{C}$, but phase pure LGdH was successfully synthesized at 90 and $120{ }^{\circ} \mathrm{C}$. The Bragg 
213 reflections of product from the $120^{\circ} \mathrm{C}$ reaction are somewhat stronger and sharper than those of the $21490^{\circ} \mathrm{C}$ analogue, implying that samples heated at the higher temperature are better crystallized and 215have larger crystallite sizes. IR spectra (Figure 3(b)) confirm the successful synthesis of LGdHs at 90 216 and $120^{\circ} \mathrm{C}$. The two spectra contain the same features, with the broad set of bands centred at ca. $2173500 \mathrm{~cm}^{-1}$ attributed to the stretching vibration of the $\mathrm{OH}$ groups from both interlayer water and the 218 hydroxide layers $[26,40]$. The absorbance at $1646 \mathrm{~cm}^{-1}$ is ascribed to the bending vibrations of the 219interlayer water molecules $\left(\delta_{\mathrm{O}-\mathrm{H}}\right)$ [57]. Bands below $1000 \mathrm{~cm}^{-1}$ correspond to the phonon vibrations of 220the structure. The peaks at 1517 and $1378 \mathrm{~cm}^{-1}$ indicate the presence of residual oleylamine, and of 221some $\mathrm{CO}_{3}{ }^{2-}$ co-intercalated with the $\mathrm{Cl}^{-}$anions, respectively.

222Table 2: Crystallite sizes of LGdH nanoparticles prepared at different temperatures, analyzed using the Scherrer equation (K $223=0.89) .{ }^{*}$ denotes occasions where no data could be obtained.

\begin{tabular}{lllll}
\hline Sample ID & \multicolumn{2}{l}{ FWHM $\left(^{\circ}\right)$} & \multicolumn{2}{c}{ Crystallite size $(\mathrm{nm})$} \\
\cline { 2 - 5 } & $(001)$ & $(220)$ & c-direction & ab-plane \\
\hline LGdH-90 & 0.339 & 0.318 & 24.6 & 27.1 \\
\hline LGdH-120 & 0.247 & $*$ & 35.3 & $*$ \\
\hline
\end{tabular}

224

225The LGdH patterns can be indexed in orthorhombic symmetry, and hence the (001) Bragg reflection 226can be applied to estimate the crystallite size along the c-axis (the thickness of the particles), while 227the (220) reflection can be used to estimate the crystallite size in the ab plane. The values estimated 228using the Scherrer equation are summarized in Table 2. The crystallite size in the c-direction is found 229to be around $25-35 \mathrm{~nm}$, with a comparable size in the ab-plane where this could be determined. 230TEM images (Figure 4) show both $\mathrm{LGdH}-90^{\circ} \mathrm{C}$ and $\mathrm{LGdH}-120^{\circ} \mathrm{C}$ to comprise small aggregates of 231platelets, with lateral sizes between around 100 and $200 \mathrm{~nm}$. The primary particle size is perhaps 232somewhat larger than the size obtained from Scherrer analysis (as would be expected given the 233assumptions involved in applying the latter), but overall the two sets of data are consistent.

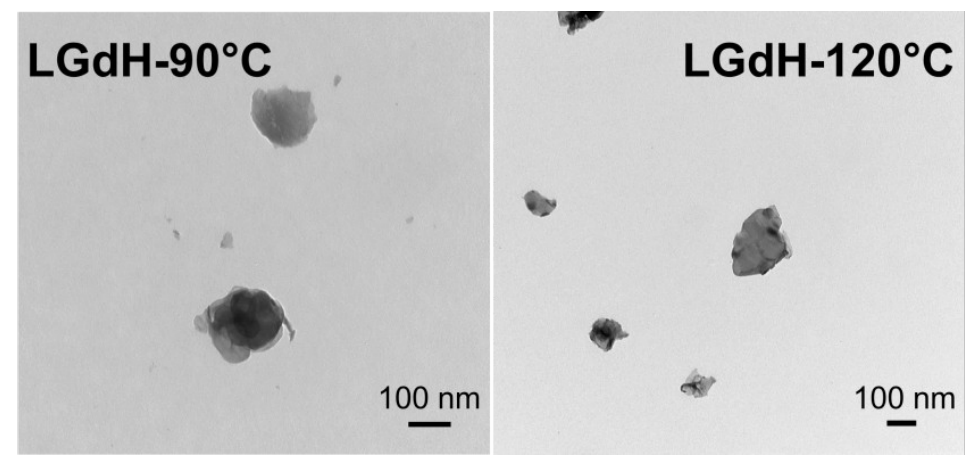

235Figure 4: TEM images of LGdH prepared at different temperatures. 


\subsection{Effect of time}

238The volume ratio of oleylamine to water and heating temperature were fixed at $1: 4$ and $90^{\circ} \mathrm{C}$, while 239the aging time was varied from 12 to $24 \mathrm{~h}$. XRD patterns of the products are given in Figure $5(\mathrm{a})$. The 240 presence of a series of $(00 /)$ basal reflections in each case indicates the formation of lamellar 241structures. The interlayer spacing of all three samples is around $8.45 \AA$, consistent with the literature 242 for $\mathrm{LGdH}-\mathrm{Cl}$ materials $[28,56]$. Scherrer analysis of the (001) and $(220)$ reflections (see $\left.120^{\circ} \mathrm{C}.\right)$ 243reveals that the crystallite size in the ab plane seems to increase with reaction time, but that in the c244diameter is largely constant, with possibly a decrease in size after reaction times longer than $12 \mathrm{~h}$. 245The IR spectra (Figure 5(b)) are analogous to those discussed previously in terms of the peak 246positions (see discussion of Figure 3(b)).
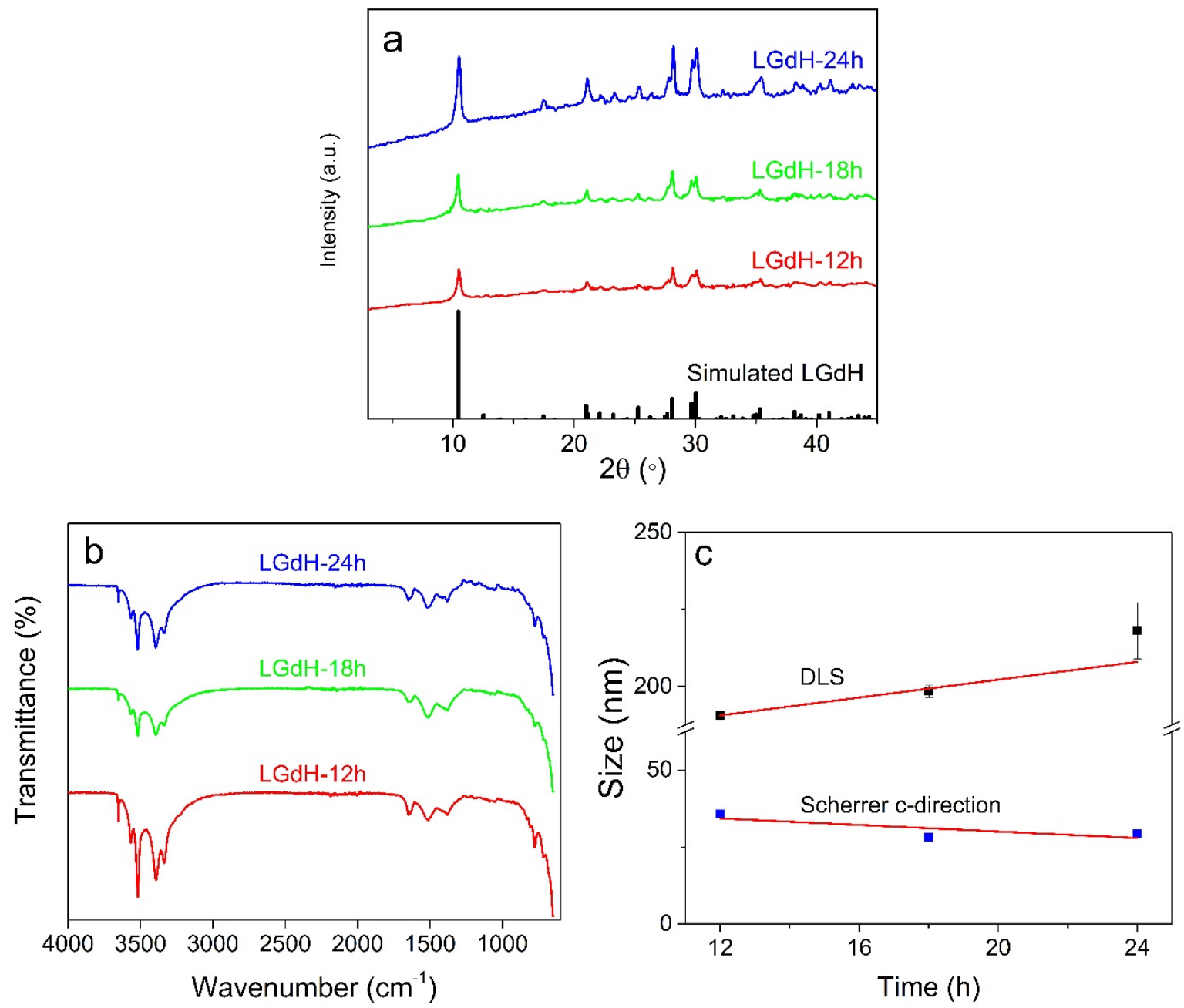

247Figure 5: Data on the LGdH products obtained after different reaction times. (a) XRD patterns; (b) IR spectra; and, (c) 248particle size data.

250DLS measurements were employed to determine the hydrodynamic particle size $\left(120^{\circ} \mathrm{C}\right.$.). There 251appears to be a trend to larger particles with longer reaction times (Figure 5(c)). TEM data are 
252presented in Figure 6. For each of LGdH-12h, LGdH-18h and LGdH-24h the samples comprise small 253aggregates of platelets, with sizes roughly in the ranges $250-500 \mathrm{~nm}, 300-700 \mathrm{~nm}$, and $250-750$ $254 \mathrm{~nm}$ respectively. These sizes are rather larger than those observed in DLS, which is ascribed to 255aggregation occurring during the TEM sample preparation process, but in general support the idea of 256larger particles forming with increased reaction times. However, the differences observed are slight, 257and overall it appears that the reaction time has little effect on the particle size.

258The LGdH-18h sample was prepared under the same conditions as the $\mathrm{LGdH}-120^{\circ} \mathrm{C}$ sample discussed 259in Section 3.2 and shown in Figure 4. The c-direction crystallite sizes, as determined through the 260Scherrer equation, are comparable for the two samples (28.2 nm for LGdH-18h, cf. $35.3 \mathrm{~nm}$ for LGdH$261120^{\circ} \mathrm{C}$ ), but the TEM particle sizes are somewhat different. This is ascribed to the propensity of the 262particles to aggregate, and the presence of different size secondary particles in the different samples 263imaged. A comparison of Figure 4 and Figure 6 reveals that the primary particle sizes are similar in 264both, and the DLS particle size for LGdH-18h agrees well with the TEM estimate of particle size for $265 \mathrm{LGdH}-120^{\circ} \mathrm{C}$.

266Table 3: Summary of particle size data for LGdH obtained after 12,18 , and $24 \mathrm{~h} .{ }^{*}$ denotes occasions where no data could be 267obtained.

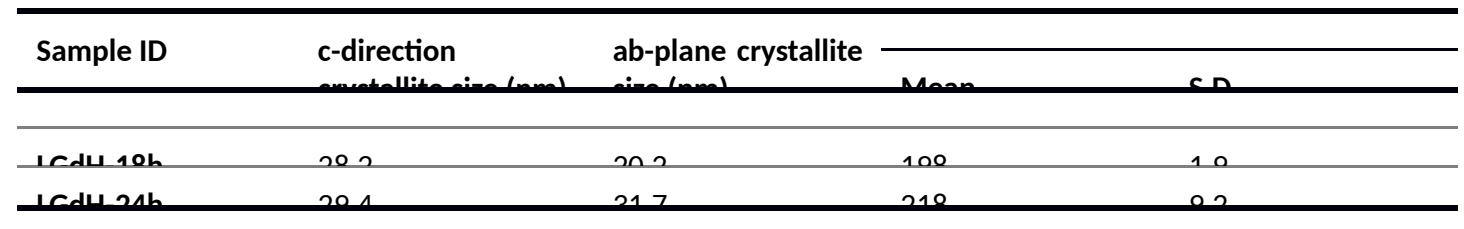

LGdH-12h

269

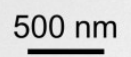

LGdH-18h

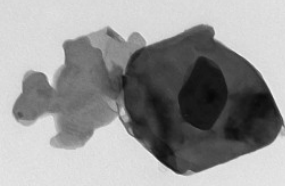

\section{LGdH-24h}

$100 \mathrm{~nm}$
$500 \mathrm{~nm}$

270Figure 6: TEM images of LGdh-12h, LGdH-18h, and LGdH-24h.

\subsection{Effect of co-surfactant}

273The literature reports that the particle size of silica nanospheres prepared by a microemulsion 274 method decreased as the amount of co-surfactant decreased [51]. Therefore, the amount of 1275 butanol used in the synthesis was varied in attempts to tune the nanoparticle size. The products' XRD 
276patterns (Figure 7(a)) all contain the characteristic basal (00/) reflections of layered LGdH particles, 277 with (001) reflections at ca. $10.5^{\circ}$ and (002) at ca. $21.1^{\circ}$. The weak reflections between the (001) and 278(002) reflections indicate the well-ordered in-plane structure of the LGdH materials. Scherrer analysis 279of the data (Table 4) does not reveal any clear relationship between the amount of co-surfactant and 280 crystallite size. $\mathrm{LGdH}-3 \mathrm{~mL}$ has the largest crystallites in the c-direction, but the non-basal reflections 281are too broad to permit measurement of their FWHM values. The other three materials have 282essentially the same crystallite size in the c-direction, and a small amount of variation in the ab 283plane, with LGdH-7mL presenting the smallest value for the latter.
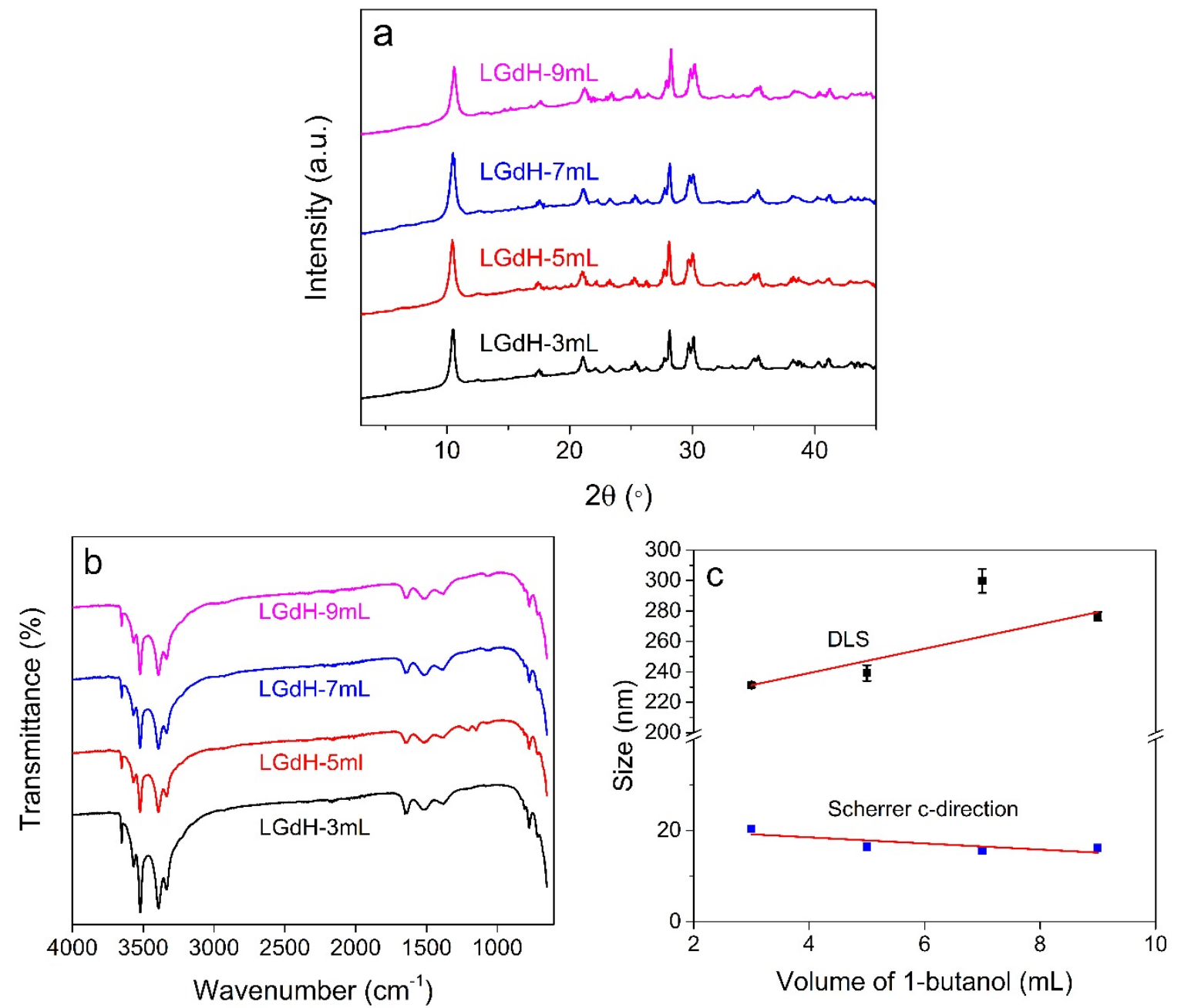

284Figure 7: The effect of changing the co-surfactant volume on LGdH synthesis. (a) XRD patterns; (b) IR spectra; and, (c) 285 particle size data.

286 The IR spectra (Figure 7(b)) show the same absorbance peaks as discussed previously, corresponding 287to $-\mathrm{OH},-\mathrm{NH}_{2}$ and $\mathrm{CO}_{3}{ }^{2-}$ and confirming the formation of $\mathrm{LGdH}$ materials. Hydrodynamic diameters 288 were measured by DLS (Table 4), and their relationship with the 1-butanol volume is presented in 289Figure 7(c). As the amount of co-surfactant increases, there is a general trend for the diameter of the 290LGdH nanoparticles to increase. This agrees with the literature findings for hollow silica nanoparticles 
291[51]. However, this trend is not completely clear, and it also apparent that there is some batch-to292batch variation in the sizes: LGdH-5mL was prepared under identical synthetic conditions to LGdH29312h, but the two have somewhat different sizes both in terms of XRD crystallite sizes and DLS particle 294sizes. Thus, although it seems that using larger amounts of co-surfactant can help to increase the 295particle size produced a little, this effect is not hugely pronounced. TEM images were obtained on 296LGdH-3mL and LGdH-5mL (data not shown), and both found to be very similar. The samples comprise 297aggragrates of platelets, with sizes in the region of $100-300 \mathrm{~nm}$.

298Table 4: Summary of the particle sizes of LGdH obtained with 3, 5, 7, and $9 \mathrm{~mL}$ of 1-butanol. * denotes that values could not 299be obtained.

\begin{tabular}{|c|c|c|c|c|}
\hline \multirow{2}{*}{ Sample ID } & \multirow{2}{*}{ C-direction } & \multirow{2}{*}{ 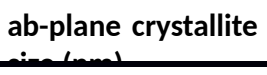 } & & \\
\hline & & & صaمMـ & nc \\
\hline ICdil Emal & $1<1$ & 111 & חחר & 工n \\
\hline $10 \mathrm{dll} 7 \mathrm{mal}$ & $15<$ & ר & n & 70 \\
\hline
\end{tabular}

300

\section{Discussion}

302The effect of the surfactant to water ratio [58], reaction temperature, reaction time and the amount 303of co-surfactant [51] used for the reverse microemulsion synthesis of LGdH have been systematically 304investigated in this work. We find that the surfactant : water ratio and the reaction temperature must 305 be carefully controlled to ensure that the desired product is formed phase-pure; the use of excessive 306amounts of surfactant ( $>1: 4$ surfactant : water ratio) or temperatures above $120^{\circ} \mathrm{C}$ lead to the 307 formation of $\mathrm{Gd}(\mathrm{OH})_{3}$, either phase-pure or as a major impurity. With a 1:4 ratio and temperatures of $308120^{\circ} \mathrm{C}$ or below, however, $\mathrm{LGdH}$ can be obtained phase-pure. The reaction time and co-surfactant 309 volume have some effect on particle size, but in all cases the sizes are found to be between 200 and $310300 \mathrm{~nm}$.

311

312It is reported that as the ratio of surfactant to water increases, the microemulsion droplets tend to 313become smaller. If the reaction is confined to the aqueous phase, as is the case here, this should lead 314to smaller particles being generated [46, 58]. LGdH particle size cannot be controlled in this way in 315the oleylamine/1-butanol system however, because gadolinium hydroxide is found to form with high 316ratios. Our findings are thus in conflict with the literature on LDH materials [47]. This is because LDHs 317are typically synthesized at ca. pH 9 - 10, while LGdHs are prepared at ca. pH 6.7 - 7.2 [54, 59]. 318Oleylamine acts both as base and surfactant, and the transformation of $\mathrm{LGdH}$ to $\mathrm{Gd}(\mathrm{OH})_{3}$ presumably 319 arises due to the increasingly strong alkaline environment as the oleylamine content of the system is 320increased. This problem does not occur with the previously-reported LDH synthesis using oleylamine 
321because of the fact that LDHs are more stable at high pHs. Therefore, the LDH nanoparticle size can 322 be controlled, and reduced to as little as $49 \mathrm{~nm}$ by tuning the surfactant to water volume ratios [47]. 323

\section{Conclusions}

325Layered gadolinium hydroxide (LGdH) nanoparticles have been successfully synthesized by employing 326a novel reverse microemulsion method. Pristine LGdH materials were obtained at temperatures 327 below $150{ }^{\circ} \mathrm{C}$ with an oleylamine : water ratio of 1:4. Control of particle size is difficult, however. 328Attempts to achieve this by using more surfactant and increasing the reaction temperature were 329 unsuccessful because of the propensity of $\mathrm{Gd}(\mathrm{OH})_{3}$ to form. It was found that at $120^{\circ} \mathrm{C}$ and below 330the temperature and the duration of heating have little effect on the size distribution, producing 331 particles around $200 \mathrm{~nm}$. Varying the amount of co-surfactant (1-butanol) used allowed the size to be 332increased from ca. $200 \mathrm{~nm}$ to $300 \mathrm{~nm}$, with use of more butanol giving larger particles. Overall, 333 microemulsions have proved to be an effective and efficient way to synthesized LGdH nanoparticles 334 of $200-300 \mathrm{~nm}$ in size, but in order to obtain more control over particle size alternative surfactants 335should be investigated.

336

\section{Acknowledgements}

338The authors gratefully thank Mr David McCarthy and Mrs Kate Keen for assistance with obtaining 339TEM images. JS and RE thank the EPSRC for the provision of PhD studentships under the Centre for 340Doctoral Training in Advanced Therapeutics \& Nanomedicines (EP/L01646X/1).

341

\section{References [Endnote has gone crazy, but will sort the refs out before} 343we resubmit!]

344 [1] M. Ranocchiari, J.A. van Bokhoven, Catalysis by metal-organic frameworks: fundamentals and 345 opportunities, Phys. Chem. Chem. Phys. 13(14) (2011) 6388-6396.

346 [2] D.O.H. Qiang Wang, Recent Advances in the Synthesis and Application of Layered Double Hydroxide (LDH) Nanosheets, Chemical reviews 12 (2012) 4124-4155.

348 [3] F. Geng, R. Ma, T. Sasaki, Anion-exchangeable layered materials based on rare-earth phosphors: unique 349 combination of rare-earth host and exchangeable anions, Acc. Chem. Res. 43(9) (2010) 1177-1185.

350 [4] S. Takagi, D.A. Tryk, H. Inoue, Photochemical energy transfer of cationic porphyrin complexes on clay 351 surface, J. Phys. Chem. B 106(21) (2002) 5455-5460.

352[5] N. Chu, Y. Sun, Y. Zhao, X. Li, G. Sun, S. Ma, X. Yang, Intercalation of organic sensitisers into layered europium 353 hydroxide and enhanced luminescence property, Dalton Trans 41(24) (2012) 7409-14. 
354 [6] Q. Qin, J. Wang, T. Zhou, Q. Zheng, L. Huang, Y. Zhang, P. Lu, A. Umar, B. Louis, Q. Wang, Impact of organic

355 interlayer anions on the CO 2 adsorption performance of $\mathrm{Mg}$-Al layered double hydroxides derived mixed oxides, Journal of Energy Chemistry 26(3) (2017) 346-353.

357 [7] V. Rives, M. del Arco, C. Martín, Intercalation of drugs in layered double hydroxides and their controlled 358 release: A review, Appl. Clay Sci. 88-89 (2014) 239-269.

359 [8] G.R. Williams, D. O'Hare, Towards understanding, control and application of layered double hydroxide 360 chemistry, Journal of Materials Chemistry 16(30) (2006) 3065.

361 [9] C. Nyambo, P. Songtipya, E. Manias, M.M. Jimenez-Gasco, C.A. Wilkie, Effect of MgAl-layered double 362 hydroxide exchanged with linear alkyl carboxylates on fire-retardancy of PMMA and PS, J. Mater. Chem. 18(40) 363 (2008) 4827-4838.

364 [10] C. Manzi-Nshuti, J.M. Hossenlopp, C.A. Wilkie, Comparative study on the flammability of polyethylene 365 modified with commercial fire retardants and a zinc aluminum oleate layered double hydroxide, Polym. Degr. 366 Stab. 94(5) (2009) 782-788.

367 [11] X. Xu, R. Lu, X. Zhao, S. Xu, X. Lei, F. Zhang, D.G. Evans, Fabrication and photocatalytic performance of a Zn 368 x Cd 1- x S solid solution prepared by sulfuration of a single layered double hydroxide precursor, Appl. Catal. B 369 102(1) (2011) 147-156.

370 371

372 373 374

375[14] Q. Wang, Z. Wu, H.H. Tay, L. Chen, Y. Liu, J. Chang, Z. Zhong, J. Luo, A. Borgna, High temperature adsorption 376 of $\mathrm{CO} 2$ on $\mathrm{Mg}-\mathrm{Al}$ hydrotalcite: effect of the charge compensating anions and the synthesis $\mathrm{pH}$, Catal. Today

384 intercalation into C 3 A hydrate phases and the role of sulfate present in cement, Cement Concrete Res. 40(1) 385 164(1) (2011) 198-203.

[15] Q. Wang, H.H. Tay, Z. Guo, L. Chen, Y. Liu, J. Chang, Z. Zhong, J. Luo, A. Borgna, Morphology and composition controllable synthesis of $\mathrm{Mg}-\mathrm{Al}-\mathrm{CO} 3$ hydrotalcites by tuning the synthesis $\mathrm{pH}$ and the $\mathrm{CO} 2$ capture capacity, Appl. Clay Sci. 55 (2012) 18-26.

[16] Q. Song, W. Liu, C.D. Bohn, R.N. Harper, E. Sivaniah, S.A. Scott, J.S. Dennis, A high performance oxygen storage material for chemical looping processes with CO 2 capture, Energy Env. Sci. 6(1) (2013) 288-298.

[17] J. Plank, D. Zhimin, H. Keller, F.v. Hössle, W. Seidl, Fundamental mechanisms for polycarboxylate (2010) 45-57.

[18] A. Alcantara, P. Aranda, M. Darder, E. Ruiz-Hitzky, Bionanocomposites based on alginate-zein/layered double hydroxide materials as drug delivery systems, J. Mater. Chem. 20(42) (2010) 9495-9504.

[19] V. Rives, M. Del Arco, C. Martin, Layered double hydroxides as drug carriers and for controlled release of non-steroidal antiinflammatory drugs (NSAIDs): a review, Journal of controlled release : official journal of the Controlled Release Society 169(1-2) (2013) 28-39. 
[20] L. Li, W. Gu, J. Chen, W. Chen, Z.P. Xu, Co-delivery of siRNAs and anti-cancer drugs using layered double hydroxide nanoparticles, Biomaterials 35(10) (2014) 3331-9.

393 [21] J.-M. Oh, S.-J. Choi, G.-E. Lee, S.-H. Han, J.-H. Choy, Inorganic drug-delivery nanovehicle conjugated with cancer-cell-specific ligand, Adv. Funct. Mater. 19(10) (2009) 1617-1624.

395[22] Z. Wang, E. Wang, L. Gao, L. Xu, Synthesis and properties of Mg2Al layered double hydroxides containing 5fluorouracil, J. Solid State Chem. 178(3) (2005) 736-741.

[23] S.A. Hindocha, L.J. McIntyre, A.M. Fogg, Precipitation synthesis of lanthanide hydroxynitrate anion exchange materials, Ln2(OH)5NO3·H2O (Ln=Y, Eu-Er), J. Solid State Chem. 182(5) (2009) 1070-1074.

[24] L.J. McIntyre, L.K. Jackson, A.M. Fogg, Ln2(OH)5NO3. x H2O ( $\mathrm{Ln}=\mathrm{Y}, \mathrm{Gd}-\mathrm{Lu}$ ): A Novel Family of Anion 400 Exchange Intercalation Hosts, Chem. Mater. 20(1) (2008) 335-340.

[25] L. Poudret, T.J. Prior, L.J. McIntyre, A.M. Fogg, Synthesis and crystal structures of new lanthanide

402 403 404 405 406 407 408 409

419 [32] Q. Zhu, J.-G. Li, C. Zhi, X. Li, X. Sun, Y. Sakka, D. Golberg, Y. Bando, Layered rare-earth hydroxides (LRHs) of $420(\mathrm{Y} 1-\mathrm{xEux}) 2(\mathrm{OH}) 5 \mathrm{NO} \cdot \mathrm{nH} 2 \mathrm{O}(\mathrm{x}=0-1)$ : Structural variations by Eu3+doping, phase conversion to oxides, and the 421 correlation of photoluminescence behaviors, Chem. Mater. 22(14) (2010) 4204-4213.

[33] B.I. Lee, S.H. Byeon, Highly enhanced photoluminescence of a rose-like hierarchical superstructure

425 [34] Q. Zhu, J.G. Li, C. Zhi, R. Ma, T. Sasaki, J.X. Xu, C.H. Liu, X.D. Li, X.D. Sun, Y. Sakka, Nanometer-thin layered 426 hydroxide platelets of $(\mathrm{YO} .95 \mathrm{Eu} 0.05) 2(\mathrm{OH}) 5 \mathrm{NO} \cdot \times \mathrm{H} 2 \mathrm{O}$ : exfoliation-free synthesis, self-assembly, and the 427 derivation of dense oriented oxide films of high transparency and greatly enhanced luminescence, J. Mater. 428

(2008) 7447-7453.

[26] W. Li, Q. Gu, F. Su, Y. Sun, G. Sun, S. Ma, X. Yang, Intercalation of azamacrocyclic crown ether into layered rare-earth hydroxide (LRH): secondary host-guest reaction and efficient heavy metal removal, Inorganic

[27] Q. Gu, Y. Sun, N. Chu, S. Ma, Z. Jia, X. Yang, Intercalation of amino acids into Eu3+-doped layered gadolinium hydroxide and quenching of Eu3+luminescence, Eur. J. Inorg. Chem. 2012(28) (2012) 4407-4412 gadolinium hydroxide: a potential MRI contrast agent, Dalton transactions (14) (2009) 2490-5.

[29] Y.-S. Yoon, B.-I. Lee, K.S. Lee, G.H. Im, S.-H. Byeon, J.H. Lee, I.S. Lee, Surface modification of exfoliated layered gadolinium hydroxide for the development of multimodal contrast agents for MRI and fluorescence imaging, Adv. Funct. Mater. 19(21) (2009) 3375-3380.

30] F. Gándara, E.G. Puebla, M. Iglesias, D.M. Proserpio, N. Snejko, M.A. Monge, Controlling the structure of arenedisulfonates toward catalytically active materials, Chem. Mater. 21(4) (2009) 655-661.

[31] Y. Xiang, X.F. Yu, D.F. He, Z. Sun, Z. Cao, Q.Q. Wang, Synthesis of highly luminescent and anionexchangeable cerium-doped layered yttrium hydroxides for sensing and photofunctional applications, Adv. Funct. Mater. 21(22) (2011) 4388-4396.

prepared by self-assembly of rare-earth hydroxocation nanosheets and polyoxomolybdate anions, Chemical communications 47(14) (2011) 4093-5. 
429 [35] X. Wu, J.G. Li, Q. Zhu, J. Li, R. Ma, T. Sasaki, X. Li, X. Sun, Y. Sakka, The effects of Gd3+ substitution on the

430 crystal structure, site symmetry, and photoluminescence of Y/Eu layered rare-earth hydroxide (LRH)

431 nanoplates, Dalton transactions 41(6) (2012) 1854-61.

432 [36] Q. Gu, N. Chu, G. Pan, G. Sun, S. Ma, X. Yang, Intercalation of diverse organic guests into layered europium 433 hydroxides - structural tuning and photoluminescence behavior, Eur. J. Inorg. Chem. 2014(3) (2014) 559-566.

434 [37] Q. Gu, F. Su, L. Ma, S. Ma, G. Sun, X. Yang, Intercalation of coumaric acids into layered rare-earth 435 hydroxides: controllable structure and photoluminescence properties, J. Mater. Chem. C 3(18) (2015) 4742436 4750.

437 [38] Q. Gu, F. Su, S. Ma, G. Sun, X. Yang, Controllable luminescence of layered rare-earth hydroxide composites 438 with a fluorescent molecule: blue emission by delamination in formamide, Chemical communications 51(13) 439 (2015) 2514-7.

440 [39] H. Kim, B.-I. Lee, H. Jeong, S.-H. Byeon, Relationship between interlayer anions and photoluminescence of 441 layered rare earth hydroxides, J. Mater. Chem. C 3(28) (2015) 7437-7445.

442 [40] T. Shen, Y. Zhang, W. Liu, Y. Tang, Novel multi-color photoluminescence emission phosphors developed by 443 layered gadolinium hydroxide via in situ intercalation with positively charged rare-earth complexes, J. Mater. Chem. C 3(8) (2015) 1807-1816.

445 [41] J. Key, J.F. Leary, Nanoparticles for multimodal in vivo imaging in nanomedicine, Int. J. Nanomedicine 9 446 (2014) 711.

447 [42] H. Dong, S.R. Du, X.Y. Zheng, G.M. Lyu, L.D. Sun, L.D. Li, P.Z. Zhang, C. Zhang, C.H. Yan, Lanthanide 448 nanoparticles: From design toward bioimaging and therapy, Chem. Rev. 115(19) (2015) 10725-815. 449 [43] D. Stefanakis, D.F. Ghanotakis, Synthesis and characterization of gadolinium nanostructured materials with 450 potential applications in magnetic resonance imaging, neutron-capture therapy and targeted drug delivery, J. 451 Nanoparticle Res. 12(4) (2010) 1285-1297.

452 [44] Y.S. Yoon, B.I. Lee, K.S. Lee, H. Heo, J.H. Lee, S.H. Byeon, I.S. Lee, Fabrication of a silica sphere with fluorescent and MR contrasting GdPO4 nanoparticles from layered gadolinium hydroxide, Chemical communications 46(21) (2010) 3654-6.

455 [45] S.S. Yoo, R. Razzak, E. Bedard, L. Guo, A.R. Shaw, R.B. Moore, W.H. Roa, Layered gadolinium-based 456 nanoparticle as a novel delivery platform for microRNA therapeutics, Nanotechnology 25(42) (2014) 425102.

457 [46] M.A. Malik, M.Y. Wani, M.A. Hashim, Microemulsion method: A novel route to synthesize organic and 458 inorganic nanomaterials, Arabian J. Chem. 5(4) (2012) 397-417.

459 [47] C.J. Wang, D. O'Hare, Synthesis of layered double hydroxide nanoparticles in a novel microemulsion, J. 460 Mater. Chem. 22(39) (2012) 21125.

461 [48] X. Zhang, K.-Y. Chan, Water-in-oil microemulsion synthesis of platinum-ruthenium nanoparticles, their $462 \quad$ characterization and electrocatalytic properties, Chem. Mater. 15(2) (2003) 451-459.

463[49] C. Murray, D.J. Norris, M.G. Bawendi, Synthesis and characterization of nearly monodisperse CdE ( $E=$ sulfur, 464 selenium, tellurium) semiconductor nanocrystallites, J. Am. Chem. Soc. 115(19) (1993) 8706-8715.

465 [50] A.J. Zarur, J.Y. Ying, Reverse microemulsion synthesis of nanostructured complex oxides for catalytic 466 combustion, Nature 403(6765) (2000) 65-67. 
467[51] C.H. Lin, J.H. Chang, Y.Q. Yeh, S.H. Wu, Y.H. Liu, C.Y. Mou, Formation of hollow silica nanospheres by reverse 468 microemulsion, Nanoscale 7(21) (2015) 9614-26.

469 [52] J.N. Coleman, M. Lotya, A. O'Neill, S.D. Bergin, P.J. King, U. Khan, K. Young, A. Gaucher, S. De, R.J. Smith, I.V. 470 Shvets, S.K. Arora, G. Stanton, H.Y. Kim, K. Lee, G.T. Kim, G.S. Duesberg, T. Hallam, J.J. Boland, J.J. Wang, J.F. 471Donegan, J.C. Grunlan, G. Moriarty, A. Shmeliov, R.J. Nicholls, J.M. Perkins, E.M. Grieveson, K. Theuwissen, D.W. 472 McComb, P.D. Nellist, V. Nicolosi, Two-dimensional nanosheets produced by liquid exfoliation of layered materials, Science 331(6017) (2011) 568-71.

474 [53] J. Rees, Synthesis, characterisation and application of layered rare-earth hydroxide nanoparticles, MChem 475 thesis, University of Liverpool, 2012, p. 61.

476 [54] F. Gandara, J. Perles, N. Snejko, M. Iglesias, B. Gomez-Lor, E. Gutierrez-Puebla, M.A. Monge, Layered rare477 earth hydroxides: a class of pillared crystalline compounds for intercalation chemistry, Angewandte Chemie 478 45(47) (2006) 7998-8001.

479 [55] F. Geng, H. Xin, Y. Matsushita, R. Ma, M. Tanaka, F. Izumi, N. Iyi, T. Sasaki, New layered rare-earth 480 hydroxides with anion-exchange properties, Chemistry 14(30) (2008) 9255-60.

481 [56] F. Geng, Y. Matsushita, R. Ma, H. Xin, M. Tanaka, F. Izumi, N. Iyi, T. Sasaki, General Synthesis and Structural 482 Evolution of a Layered Family of $\mathrm{Ln} 8(\mathrm{OH}) 20 \mathrm{Cl} 4 \cdot \mathrm{n} \mathrm{H} 2 \mathrm{O}(\mathrm{Ln}=\mathrm{Nd}, \mathrm{Sm}, \mathrm{Eu}, \mathrm{Gd}, \mathrm{Tb}$, Dy, Ho, Er, Tm, and Y), J. Am. 483 Chem. Soc. 130(48) (2008) 16344-16350.

484 [57] Z.P. Xu, G.Q. Lu, Hydrothermal synthesis of layered double hydroxides (LDHs) from mixed MgO and Al2O3: 485 LDH formation mechanism, Chem. Mater. 17(5) (2005) 1055-1062.

486 [58] G. Hu, N. Wang, D. O'Hare, J. Davis, Synthesis of magnesium aluminium layered double hydroxides in 487 reverse microemulsions, J. Mater. Chem. 17(21) (2007) 2257.

[59] K.-H. Lee, S.-H. Byeon, Extended Members of the Layered Rare-Earth Hydroxide Family, $\mathrm{RE} 2(\mathrm{OH}) 5 \mathrm{NO} 3 \cdot \mathrm{nH} 2 \mathrm{O}(\mathrm{RE}=\mathrm{Sm}, \mathrm{Eu}$, and Gd): Synthesis and Anion-Exchange Behavior, Eur. J. Inorg. Chem. 